\title{
Multi-sensor Information Fusion Method Based on BP Neural Network
}

\author{
http://dx.doi.org/10.3991/ijoe.v12i05.5738 \\ Lin Liandong \\ HeiLongJiang University, HeiLongJiang, Harbin China
}

\begin{abstract}
This study aims to solve the problem of multisensor information fusion, which is a key issue in the multisensor system development. The main innovation of this study is to propose a novel multi-sensor information fusion algorithm based on back propagation neural network and Bayesian inference. In the proposed algorithm, a triple is defined to represent a probability space; thereafter, the Bayesian inference is used to estimate the posterior expectation. Finally, we construct a simulation environment to test the performance of the proposed algorithm. Experimental results demonstrate that the proposed algorithm can significantly enhance the accuracy of temperature detection after fusing the data obtained from different sensors.
\end{abstract}

Index Terms-Information fusion, BP neural network, Bayesian inference, Probability space

\section{INTRODUCTION}

The rapid development of science and technology has immensely improved the performance of sensors and the emergence of numerous complex applications in sensor systems. From the beginning of the 20th century, information provided by a single sensor can no longer meet human requirements [1-2]. Therefore, using multiple sensors is necessary to provide observational data and services for real-time target detection and recognition. In the multi-sensor system, the diversity and immense amount of information, as well as the complexity of the relational information, has substantially exceeded the human brain's information processing capabilities [3].

Multi-sensor system provides a purposeful description of the environment [4-5]. Fusing several types of information may improve the ability of the intelligent system; however, achieving this level is impossible for one sensor. Thus, multi-sensor fusion refers to emerging technologies in the information science, computer science, and automatic science domains, among others [6-8].

Information fusion technology refers to a new field in information science. In the $1970 \mathrm{~s}$, the continuous progress of science and technology had resulted in the emergence of numerous multi-sensor systems, particularly with the rapid development of microelectronics, signal detection and processing, computers, and network communication, among others. [9]. In the past 20 years, the multi-sensor information fusion technology has received considerable attention. Information fusion refers to a novel approach in information processing to deal with the issue of multiple sensors system. Information fusion is also called multisource correlation [10]. Currently, providing a unified definition of information fusion is considerably difficult, thereby resulting in the universality and diversity of research on information fusion.

The rest of this paper is organized as follows. Section 2 presents the literature review of information fusion applications. Section 3 provides the basic theory of back propagation neural network (BPNN). Section 4 proposes a novel multi-sensor information fusion algorithm based on BPNN and Bayesian inference. Section 5 presents the experiments we conducted to test the effectiveness of the proposed algorithm. Finally, Section 6 concludes this study.

\section{LITERATURE REVIEW}

Information fusion is used to merge information from heterogeneous sources with different information representations. This method has been maximized in data mining for both unstructured and semi-structured data types. In particular, information fusion denotes textual representations of knowledge with rich-media contents. In this section, we present the related studies on this concept.

$\mathrm{Xu}$ et al. utilized sensory information fusion technology to minimize diagnostic uncertainty and computational complexity. In particular, this study developed a sensory information fusion-based diagnostic system comprising a dynamic sensor selection integrating sensory information [11].

Tang et al. proposed a rule acquisition method for triadic decision contexts, which is an information fusion technology for decision-making analysis of multi-source data. They also constructed a knowledge reduction framework to model knowledge discovery [12].

Zhang et al. aimed to fuse micro electro mechanical system inertial measurement unit (MEMS-IMU) information with optical flow to enhance the MEMS performance. These researchers also presented an image coordinate of optical flow to be transformed to a single body for the vehicle attitude computation [13].

Wang et al. developed the total orders of hesitant fuzzy elements (HFEs) for multi-criteria decision-making, as well as utilized ordered weighted aggregation operators to fuse information. Furthermore, admissible orders are able to enhance the existing partial orders of HFEs and can be generated by a set of special functions [14].

$\mathrm{Li}$ et al. proposed a modeling-free solution to infer the state directly from observations, as well as developed a dynamic state estimation to describe the system as a hidden Markov process. Thereafter, their study maximized a recursive estimator to effectively fuse the time-series data [15]. 
$\mathrm{Wu}$ et al. proposed an information fusion-based detection and identification approach to detect and identify distributed attacks. The main innovation of their study is applying principal component analysis to design detection and identification approaches [16].

Apart from the aforementioned studies, information fusion, such as wheat varieties classification [17], rule-based Gaussian-shaped fuzzy control inference system [18], intuitionistic fuzzy decision making [19], and gait-based re-identification and recognition [20], can also be utilized in other fields. In the current research, we study the information fusion problem in multi-sensor environments using BPNN.

\section{BASIC THEORY OF BPNN}

Artificial neural network (ANN) refers to a nonlinear system that simulates the cerebrum information processing algorithm [21]. ANN has powerful distributed information storage, parallel processing, and adaptive learning capability [22]. Using the BP definition, the multilayer neural network model is defined as BPNN that is designed based on neural network theory [23]. BPNN is characterized for its simple structure and technical maturity [24-25]; hence, this model has been extensively utilized in pattern recognition, intelligent control, and state prediction, among others [26-27].

BPNN comprises three parts, namely, input, hidden, and output layers. Figure 1 provides the BPNN architecture to illustrate its structure.

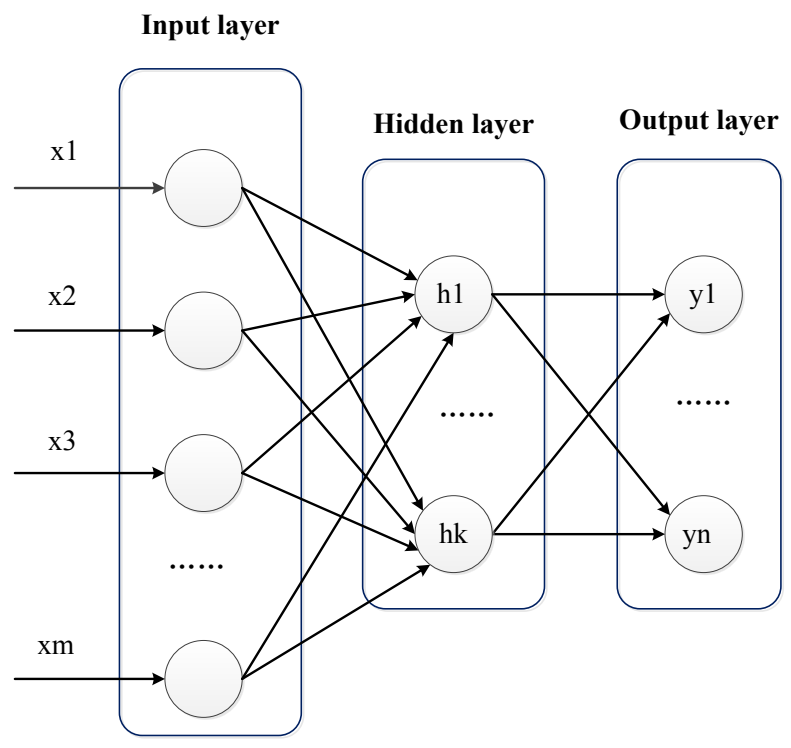

Figure 1. Architecture of the BP neural network

The continuous nonlinear excitation functions in BPNN should be defined in advance.

$$
F\left(n e t_{j k}\right)=\frac{1}{\left(1+e^{-N e t_{k j}}\right)},
$$

where $n e t_{j k}$ is computed by the following equation:

$$
n e t_{j k}=\sum_{i=N} w_{j i} \cdot O T_{k i}+\delta_{j}
$$

$$
O T_{k i}=\left(1+\exp \left(-\sum_{i=1}^{N} w_{j i} \cdot O T_{k i}-\delta_{j}\right)\right)^{-1}
$$

Furthermore, parameter $\omega_{k j}$ in the output layer can be estimated as follows:

$$
\omega_{k j}=\left(t_{k j}-O T_{k j}\right) \cdot O T_{k j} \cdot\left(1-O T_{k j}\right) .
$$

Different from the output layer, parameters of the hidden layer are calculated as follows:

$$
\omega_{k j}=O T_{k j} \cdot\left(1-O T_{k j}\right) \cdot \sum_{m=1}^{M} \omega_{k m} \cdot v_{u j} .
$$

In BPNN, the outputs of each layer are transmitted to their neurons in the next layer. That is, BPNN is able to hold a bias neuron to produce constant outputs without any inputs. From the preceding process, BPNN is built up via minimizing errors in the neural networks, which is similar to the neural network.

\section{MULTI-SENSOR INFORMATION FUSION USING BPNN AND BAYESIAN INFERENCE}

Suppose that $X=\left(x_{1}, x_{2}, \mathrm{~L}, x_{M}\right)^{T}$ belongs to $D_{x}=D_{x 1} \times D_{x 2} \times \mathrm{L} \times D_{x M}$, and the objective of statistical inference is to lower an unknown information from the observation sequence $y=\left(y_{1}, y_{2}, \mathrm{~L}, y_{N}\right)^{T}$. Assume that the triple $(\Omega, F, P)$ refers to a probability space, where $\Omega, F, P$ denote the sample space, $\sigma$ field, and probability measure, respectively.

In general, Bayesian inference aims to calculate the posterior expectation. This process can be described as follows:

$$
E[h(X) \mid y]=\int_{D x} h(x) \pi(x \mid y) d x
$$

where $X: \Omega \rightarrow D_{x} \subset R^{M}$ is a random vector and $\pi(x \mid y)$ refers to an updated probability density. Furthermore, information fusion based on the posterior density is represented as follows:

$$
E[h(X) \mid y]=\frac{E[h(x) L(x)]}{Z}
$$

where $L(x)=f(y \mid x)$ is a proposed likelihood function to describe the conditional density $f(y \mid x)$, and $Z$ is the model evidence that can normalize the posterior density.

$$
Z=\int_{D x} L(x) \pi(x) d x
$$

The main idea of our study is to utilize the multi-sensor data fusion method in the decision level to integrate information obtained from multiple neural networks. Figure 2 illustrates the flowchart of the proposed multi-sensor information fusion method. 


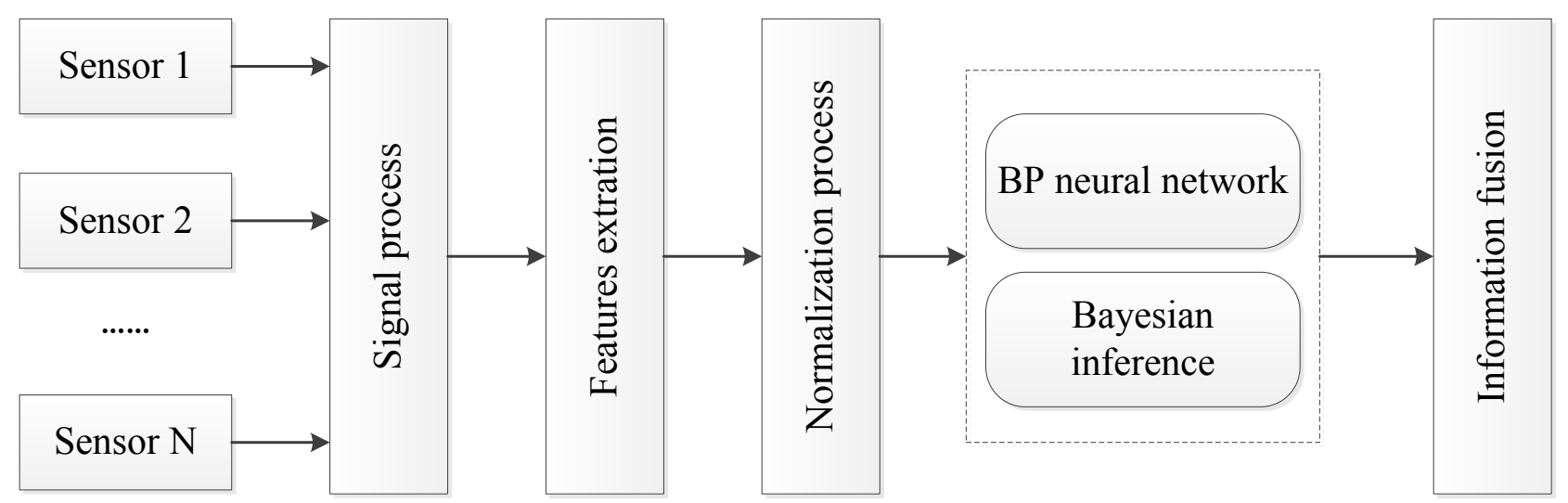

Figure 2. Flowchart of the proposed multi-sensor information fusion method.

To promote the accuracy of the information fusion, we should modify the standard Bayesian inference model. In the Bayesian inference, we assume that $H_{1}, H_{2}, \mathrm{~L}, H_{i}$ represents the hypothesis that refers to an observation $O$. Thereafter, the posterior probability of $H_{k}$ is defined as follows:

$$
P\left(H_{k} \mid E\right)=\frac{P\left(E \mid H_{k}\right) \times P\left(H_{k}\right)}{\sum_{j=1}^{N} P\left(E \mid H_{j}\right) \times P\left(H_{j}\right)}
$$

subject to

$$
\sum_{j=1}^{N} P\left(H_{j}\right)=1
$$

where $P\left(H_{j}\right)$ means the priori probability of the condition that $H_{j}$ is true and $P\left(E \mid H_{j}\right)$ refers to the observation evidence $O$ with the condition that $H_{j}$ is true. To model the multi-sensor information fusion process, each individual neural network is defined as follows:

$$
y_{t}=y_{t}^{h}\left[\begin{array}{l}
y(t-1), \mathrm{L}, y\left(t-n_{o}\right) \\
z(t-1), \mathrm{L}, z\left(t-m_{i}\right)
\end{array}\right]+e r r_{t}^{h},
$$

where symbol $y_{t}$ refers to the real process output at the time slot $t, z$ means the process input, function $y_{t}^{h}()$ is designed to forecast from the $h^{\text {th }}$ neural network at $t$, and $\mathrm{err}_{t}^{h}$ is the error of the $h^{\text {th }}$ neural network at $t$. In particular, $n_{o}$ and $m_{i}$ denote the time lags extracted from output and input, respectively.

Based on the aforementioned definitions, the proposed model can fuse information from multi-sensor as follows:

$$
\hat{y}_{t}=\sum_{i=1}^{n}\left(\delta_{t}^{i} \cdot y_{t}^{i}\right)
$$

where $\hat{y}_{t}$ refers to the information fusion results. In particular, parameter $\delta_{t}^{i}$ means the weight used to fuse the $i^{\text {th }}$ neural network at time $t$.

\section{EXPERIMENT}

In the current experiment, the numbers of input and output neurons are set to 15 and 3, respectively; we do not fix the number of neurons in one hidden layer. Before solving the multi-sensor information fusion task, we should utilize BPNN to train samples; error rate rapidly decreases to $10^{-5}$.

Thereafter, we construct a simulation environment to test the performance of the proposed information fusion algorithm. We suppose that the average temperature of the target is $60^{\circ} \mathrm{C}$, and three different temperature sensors are used. In particular, the detection error rates of these three sensors are $\sigma_{1}^{2}=1, \sigma_{2}^{2}=2$, and $\sigma_{3}^{2}=3$. Thereafter, we randomly generate three observation sequences $\left(X_{1}, X_{2}\right.$, and $X_{3}$ ) from these three sensors. We assume that the number of observations is set to $n$ and the observation value sequence $X_{i}$ is equal to $\left\{x_{i 1}, x_{i 2}, \mathrm{~L}, x_{i n}\right\}$. First, the observation sequences of each sensor are fused in advance and the fusing results are represented as $\left\{\hat{x}_{1}, \hat{x}_{2}, \hat{x}_{3}\right\}$. Second, the final information fusion results (denoted as $\hat{x}$ ) are obtained using the proposed algorithm. The numbers of observations $(N)$ are set as 20, 50, 90, and 120. Figures 3 to 6 illustrate the observation distributions under different settings. 
PAPER

Multi-SENSOR INFORMATION Fusion METHOD BASED ON BP NEURAL NETWORK

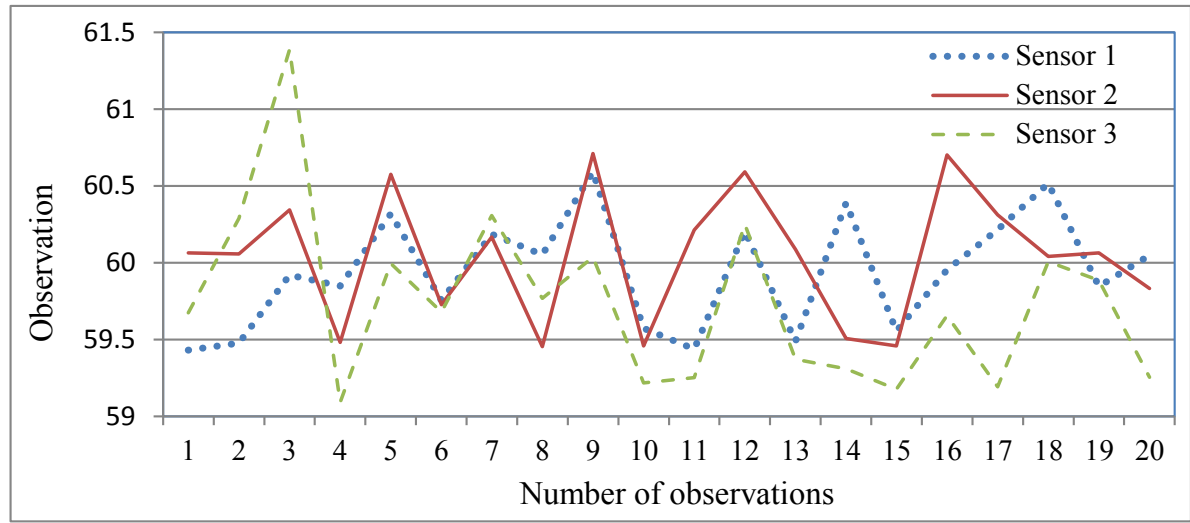

Figure 3. Observation values for $\mathrm{N}=20$

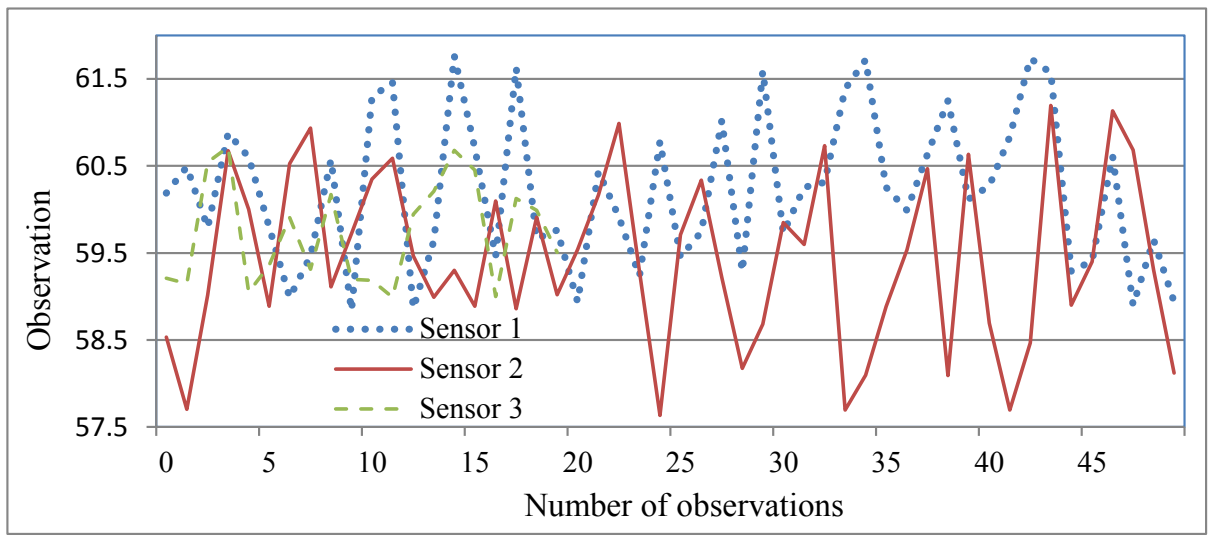

Figure 4. Observation values for $\mathrm{N}=50$

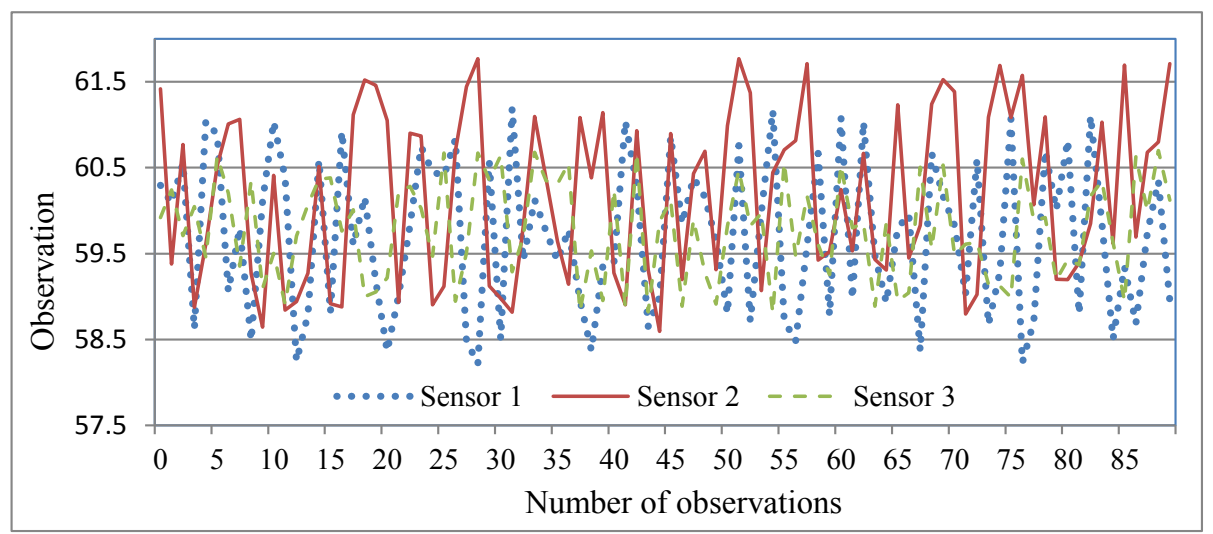

Figure 5. Observation values for $\mathrm{N}=90$

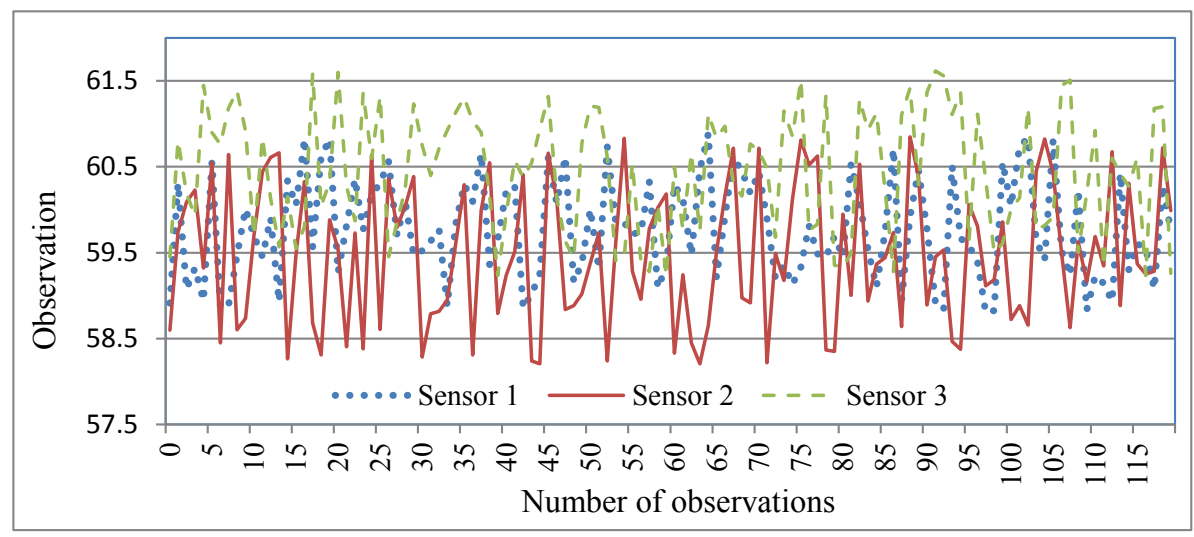

Figure 6. Observation values for $\mathrm{N}=120$ 
Thereafter, we maximize the proposed multi-sensor information fusion algorithm to fuse the aforementioned observation sequences obtained from the different sensors. Table 1 provides the final information fusion results (denoted as $\hat{x}$ ).

TABLE I.

FUSION RESULTS OBTAINED USING THE PROPOSED ALGORITHM

\begin{tabular}{c|c|c|c|c}
\hline $\begin{array}{c}\text { Number of obser- } \\
\text { vations }\end{array}$ & $\hat{x}_{1}$ & $\hat{x}_{2}$ & $\hat{x}_{3}$ & $\hat{x}$ \\
\hline 20 & 59.939 & 60.066 & 59.739 & 59.941 \\
\hline 50 & 60.222 & 59.429 & 59.804 & 60.042 \\
\hline 90 & 59.712 & 60.202 & 59.777 & 59.962 \\
\hline 120 & 59.771 & 59.485 & 60.44 & 60.033 \\
\hline
\end{tabular}

Table 1 shows that the proposed multi-sensor information fusion algorithm accurately detects the temperature. Moreover, we observe that the temperature detected by the proposed information fusion algorithm is considerably close to the actual temperature with the number of observations increasing. This result is founded on the fact that further observations can effectively reduce the negative influences of noisy information. The quality of information fusion is also enhanced significantly.

\section{CONCLUSION}

In this study, we focus on the problem of multi-sensor information fusion and maximize the data obtained from the different sensors. In the proposed algorithm, data extracted from the sensors are processed through three steps, namely, 1) signal process, 2) features extraction, and 3) normalization process. Thereafter, the multi-sensor information fusion is performed by BPNN and Bayesian inference. The experimental results demonstrate that the quality of information fusion is enhanced significantly by the proposed algorithm.

\section{REFERENCES}

[1] Rawat Sarvesh, Rawat Surabhi, "Multi-sensor Data Fusion by a Hybrid Methodology - A Comparative Study", Computers in Industry, 2016, vol. 75 , pp. 27-34. http://dx.doi.org/10.1016/j.com pind.2015.10.012

[2] Voelker Christoph, Shokouhi Parisa, "Clustering Based Multi Sensor Data Fusion for Honeycomb Detection in Concrete", Journal of Nondestructive Evaluation, 2015, vol. 34, no. 4, Article No 32.

[3] Zhang Ping, Liu Xin, Du Guanglong, Liang Bin, Wang Xueqian, "A Markerless Human-Manipulators Interface Using MultiSensors", Industrial Robot-An International Journal, 2015, vol. 42, no. 6, pp. 544-553. http://dx.doi.org/10.1108/IR-03-2015-0057

[4] Geng Hang, Liang Yan, Yang Feng, Xu Linfeng, Pan Quan, "The Joint Optimal Filtering and Fault Detection for Multi-Rate Sensor Fusion under Unknown Inputs", Information Fusion, 2016, vol. 29, pp. 57-67. http://dx.doi.org/10.1016/j.inffus.2015.10.003

[5] Telling J, Flower V J B, Carn S A, "A Multi-Sensor Satellite Assessment of SO2 Emissions from the 2012-13 Eruption of Plosky Tolbachik Volcano, Kamchatka”, Journal of Volcanology and Geothermal Research, 2015, 307: 98-106. http://dx.doi.org/10.1016/j.jvolgeores.2015.07.010

[6] Hullo Jean-Francois, Thibault Guillaume, Boucheny Christian, Dory Fabien, Mas Arnaud, "Multi-Sensor As-Built Models of Complex Industrial Architectures", Remote Sensing, 2015, vol. 7, no. 12, pp. 16339-16362. http://dx.doi.org/10.3390/rs71215827

[7] Holzer N, Vijay S, Yao T, Xu B, Buchroithner M, Bolch T, "Four Decades of Glacier Variations at Muztagh Ata (Eastern Pamir): A Multi-sensor Study including Hexagon KH-9 and Pleiades Data",
Cryosphere, 2015, vol.9, no.6, pp. 2071-2088. http://dx.doi.org/10.5194/tc-9-2071-2015

[8] Meng Lingkui, Dong Ting, Zhang Wen, "Drought Monitoring Using an Integrated Drought Condition Index (IDCI) derived from Multi-sensor Remote Sensing Data", Natural Hazards, 2016, vol. 80, no. 2, pp. 1135-1152. http://dx.doi.org/10.1007/s11069-0152014-1

[9] Rahman A F R, Fairhurst M C. "Multiple expert classification: a new methodology for parallel decision fusion", International Journal on Document Analysis and Recognition, 2000, vol. 3, no. 1, pp. 40-55. http://dx.doi.org/10.1007/PL00013554

[10] Rodriguez R M, Bedregal B, Bustince H, Dong Y C, Farhadinia B, Kahraman C, Martinez L, Torra V, Xu Y J, Xu Z S, Herrera F, "A Position and Perspective Analysis of Hesitant Fuzzy Sets on Information Fusion in Decision Making towards High Quality Progress", Information Fusion, 2016, vol. 29, pp. 89-97. http://dx.doi.org/10.1016/j.inffus.2015.11.004

[11] Xu Lei, Xu Jiuping, "Sensory Information Fusion-Based Fault Diagnostics for Complex Electronic Systems", Proceedings of the Institution of Mechanical Engineers Part O-Journal of Risk and Reliability, 2016, vol. 230, no. 1, pp. 109-119. http://dx.doi.org/10.1177/1748006X15599125

[12] Tang Yaqiang, Fan Min, Li Jinhai, "An Information Fusion Technology for Triadic Decision Contexts", International Journal of Machine Learning and Cybernetics, 2016, vol. 7, no. 1, pp. 1324. http://dx.doi.org/10.1007/s13042-015-0411-0

[13] Zhang Ling, Xiong Zhi, Lai Jizhou, Liu Jianye, "Optical Flowaided Navigation for UAV: A Novel Information Fusion of Integrated MEMS Navigation System”, Optik, 2016, vol. 127, no. 1, pp. 447-451. http://dx.doi.org/10.1016/j.ijleo.2015.10.092

[14] Celik E, Gul M, Aydin N, et al. "A comprehensive review of multi criteria decision making approaches based on interval type-2 fuzzy sets", Knowledge-Based Systems, 2015, vol. 85, pp. 329341. http://dx.doi.org/10.1016/j.knosys.2015.06.004

[15] Li Tiancheng, Corchado Juan M, Bajo Javier, Sun Shudong, De Paz Juan F, "Effectiveness of Bayesian Filters: An Information Fusion Perspective", Information Sciences, 2016, vol. 329, pp. 670-689. http://dx.doi.org/10.1016/j.ins.2015.09.041

[16] Wu Hao, Dang,Xianglei, Wang Lidong, He Longtao, "Information Fusion-Based Method for Distributed Domain Name System Cache Poisoning Attack Detection and Identification", Iet Information Security, 2016, vol. 10, no. 1, pp. 37-44. http://dx.doi.org/10.1049/iet-ifs.2014.0386

[17] Dong Gao, Guo Jian, Wang Cheng, Chen Zi-long, Zheng Ling, Zhu Da-zhou, "The Classification of Wheat Varieties Based on Near Infrared Hyperspectral Imaging and Information Fusion", Spectroscopy and Spectral Analysis, 2015, vol. 35, no. 12, pp. 3369-3374.

[18] Li Zairan, He Ting, Cao Luying, Wu Tunhua, McCauley Pamela, Balas Valentina E, Shi Fuqian, "Multi-Source Information Fusion Model in Rule-Based Gaussian-Shaped Fuzzy Control Inference System Incorporating Gaussian Density Function", Journal of Intelligent \& Fuzzy Systems, 2015, vol. 29, no. 6, pp. 2335-2344. http://dx.doi.org/10.3233/IFS-151932

[19] Xia M, Xu Z. "Hesitant fuzzy information aggregation in decision making”. International journal of approximate reasoning, 2011, vol. 52, no. 3, pp. 395-407. http://dx.doi.org/10.1016/j.ijar.2010. 09.002

[20] Chattopadhyay Pratik, Sural Shamik, Mukherjee Jayanta, "Information Fusion from Multiple Cameras for Gait-Based ReIdentification and Recognition", IET Image Processing, 2015, vol. 9, no. 11, pp. 969-976. http://dx.doi.org/10.1049/iet-ipr.2014.0773

\section{AUTHOR}

Lin Liandong is with the Electronic Engineering College of HeiLongJiang University, Nangang District of Harbin City University Road 74, HeiLongJiang, Harbin 150080, China (e-mail: 86265029@qq.com)

Submitted 29 March 2016. Published as resubmitted by the author 08 May 2016. 